6. Nichols WL, Hultin MB, James AH, et al. The Diagnosis, Evaluation, and Management of von Willebrand Disease. National Heart, Lung, and Blood Institute. NIH Pub. No. 08-5832. Hemophilia. 2008;14:171-232.

7. Mannucci PM, Franchini M, Castaman G, et al. Evidence-based recommendations on the treatment of von Willebrand disease in Italy. Blood Transfusion. 2007;7:117-126.

8. Hazendonk H, Heijdra JM, de Jager N, et al. Analysis of current perioperative management with Humate $\mathrm{P}^{\circledR}$ in von Willebrand disease: identifying the need for personalized treatment. Haemophilia. 2018;24:460-470.

9. Lethagen S, Kyrle PA, Castaman G, et al. The humate P surgical study group. Von Willebrand factor/factor VIII concentrate (humate P) dosing based on pharmacokinetics: a prospective multicenter trial in elective surgery. J Thromb Haemost. 2007;5:1420-1430.

10. Rivard GE, Aledort L, Alphanate surgical investigators. Efficacy of factor VIII/von Willebrand factor concentrate Alphanate in preventing excessive bleeding during surgery in subjects with von Willebrand disease. Haemophilia. 2008;14:271-275.

11. Nitu-Whalley IC, Griffioen A, Harrington C, Lee CA. Retrospective review of the management of elective surgery with desmopressin and clotting factor concentrates in patients with von Willebrand disease. Am J Hematol. 2001;66:280-284.

12. Thompson AR, Gill JC, Ewenstein BM, et al. Successful treatment for patients with von Willebrand disease undergoing urgent surgery using factor VIII/VWF concentrate $\left(\right.$ Humate- $\left.\mathrm{P}^{\circledR}\right)$. Haemophilia. 2004;10:42-51.

13. Mannucci P, Kyrle P, Schulman S, et al. Prophylactic efficacy and pharmacokinetically guided dosing of a von Willebrand factor/factor VIII concentrate in adults and children with von Willebrand's disease undergoing elective surgery: a pooled and comparative analysis of data from USA and European Union clinical trials. Blood Transfusion. 2011;11:533-540.

\title{
Emicizumab prophylaxis to facilitate anticoagulant therapy for management of intra-atrial thrombosis in severe haemophilia with an inhibitor
}

A 6-year-old boy with severe haemophilia $A$ and high-titre inhibitor (peak 41.8 Bethesda units [BU]), undergoing immune tolerance induction (ITI) with high dose ( 120 units $/ \mathrm{kg} / \mathrm{d})$ extended half-life recombinant factor VIII (FVIII) (recombinant Fc fusion protein) developed central venous access device (CVAD)-associated bacteremia. For prophylaxis while on ITI, he had been receiving $1-2 \times$ daily activated prothrombin complex concentrate (aPCC, 100 units $/ \mathrm{kg} /$ dose) prophylaxis, with recombinant factor VIla (rFVIla, $200 \mu \mathrm{g} / \mathrm{kg} / \mathrm{dose}$ ) for breakthrough bleeds via a port CVAD. During evaluation for his bacteremia, an echocardiogram revealed a "mobile target" at the end of his port in the right atrium (Figure 1 for clinical timeline). His port was removed, and a peripherally inserted central catheter (PICC) was placed.

A new port was placed, and 6 months later, he was admitted with recurrent bacteremia. A repeat echocardiogram demonstrated the thrombus, which measured $1.7 \times 1.4 \mathrm{~cm}$ in size. Follow-up echocardiograms demonstrated the mass to be stable in size. During the admission for port removal, he was found to have a negative Bethesda titre and sufficient FVIII recovery, but did not meet criteria for tolerance given continued short half-life. Given this, and concerns for thrombus exacerbation, aPCC was discontinued. A follow-up echocardiogram 3 months later demonstrated decreasing thrombus size to $0.6 \times 0.4 \mathrm{~cm}$. Given partial reabsorption and lack of symptoms, the decision was made to continue observation with scheduled echocardiograms. Six months later, he developed recurrent hemarthroses into his left ankle in the context of an inhibitor titre of $17.5 \mathrm{BU}$ and prophylactic daily aPCC was resumed. Three months later, echocardiogram showed a marginal increase in thrombus size. He was monitored with serial echocardiograms over the next year with no change.
He continued to have intermittent hemarthroses and required one hospitalization for intensive infusions with alternating aPCC (85 units/ $\mathrm{kg} /$ dose) and rFVIla (120 $\mathrm{\mu g} / \mathrm{kg} /$ dose) every 6 hours. An echocardiogram demonstrated significant enlargement of the thrombus to $2.4 \times 1.4 \mathrm{~cm}$, with new extension towards the tricuspid valve annulus, and $9-10 \mathrm{~mm} \mathrm{Hg}$ gradient across the tricuspid valve. The patient reported a week long history of intermittent fatigue, dizziness and diaphoresis with minimal exertion. The severity of his symptomatic obstruction to right ventricular inflow and large mobile thrombus was an indication for surgical thrombectomy on cardiopulmonary bypass (CPB). Percutaneous options for intervention were considered and not thought to be viable. He was hospitalized, and all infusions through his port discontinued. It was presumed that the thrombus progression was likely attributable to continued use of bypassing agent therapy. We hypothesized that prophylaxis with emicizumab would allow haemostatic control of bleeding yet result in a less procoagulant balance compared to prior bypassing therapy prophylaxis. He began treatment at the standard loading dose of $3 \mathrm{mg} / \mathrm{kg}$ subcutaneously weekly for 4 weeks followed by maintenance dosing of $1.5 \mathrm{mg} / \mathrm{kg}$ weekly. Upon transition to maintenance dosing, a repeat echocardiogram revealed no change in thrombus size. Therefore, formal anticoagulation was initiated with twice daily low molecular weight heparin (LMWH) at a goal anti-Xa of $0.3-0.5 \mathrm{IU} / \mathrm{mL}$ (typical goals $0.1-0.3 \mathrm{IU} / \mathrm{mL}$ prophylactic dosing, and $0.5-1 \mathrm{IU} / \mathrm{mL}$ treatment dosing) to first evaluate his bleeding risk. After 4 days, there was no change on repeat echocardiogram yet he was not having any bleeding manifestations, so his anti-Xa target was increased to $0.5-0.7 \mathrm{IU} / \mathrm{mL}$. Following 2 weeks of anticoagulation, the thrombus appeared slightly smaller in size and 


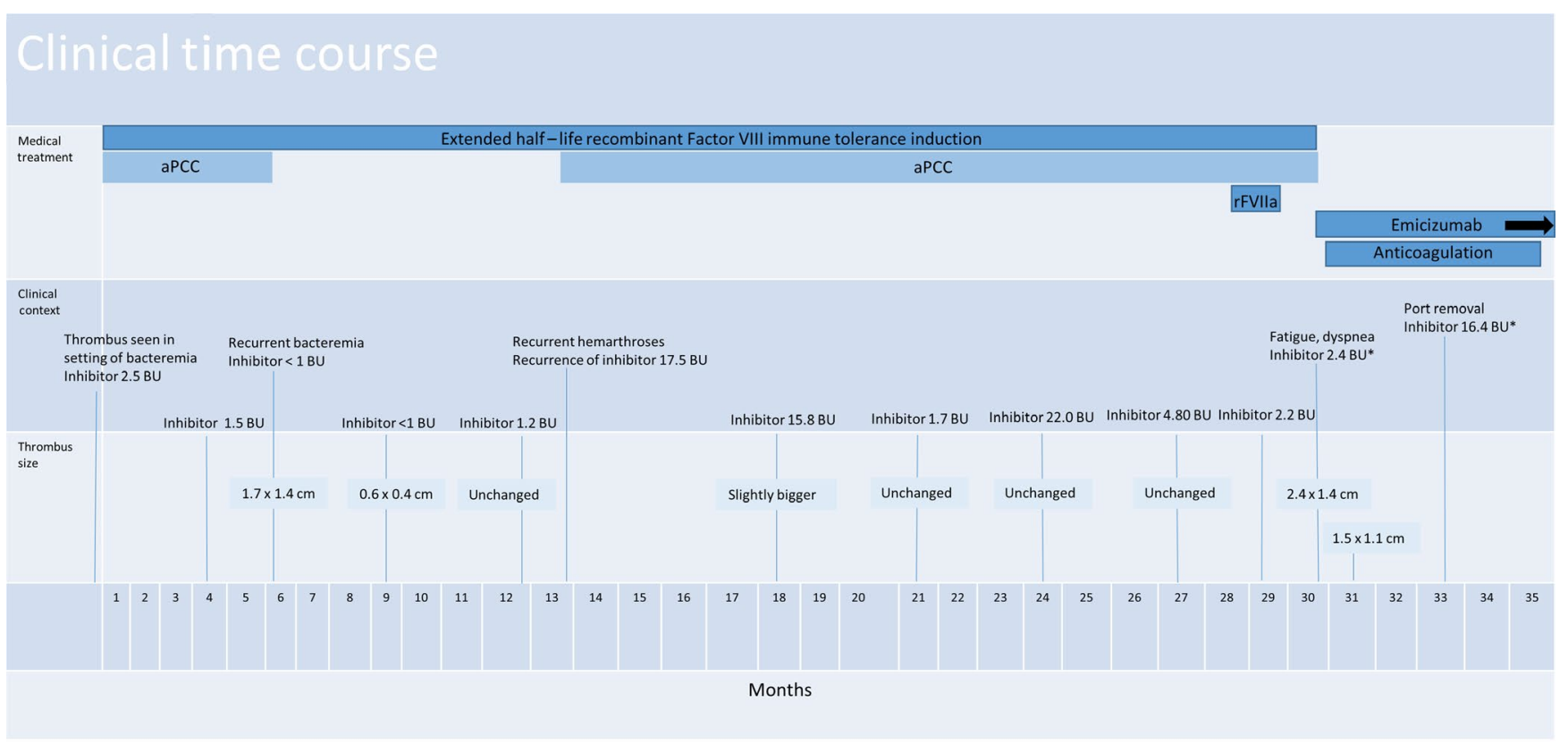

* Bethesda assay performed with bovine chromogenic FVIII

FIGURE 1 Clinical time course

increasingly organized, thus this management plan was continued. The patient continued to tolerate anticoagulation well without significant bleeding symptoms. He developed one spontaneous haematoma on his upper arm but otherwise had only expected bruises at injection sites. Following 2 months of anticoagulation, a repeat echocardiogram demonstrated significant improvement. The thrombus had decreased in size to $1.5 \times 1.1 \mathrm{~cm}$ and was less mobile, the mean inflow gradient had decreased to 3-5 $\mathrm{mm} \mathrm{Hg}$ from $12 \mathrm{~mm} \mathrm{Hg}$ previously, and there was improved motion of tricuspid valve leaflets. Additionally, he had symptomatic improvement with decreased heart rate and improved activity tolerance. Following 3 months of anticoagulation, his port was removed. Prior to removal, he continued emicizumab (but received a one-time higher dose of $3 \mathrm{mg} / \mathrm{kg}$ prior to surgery under the rationale that this may provide improved haemostasis) and his LMWH was held for 24 hours prior to the procedure. He did not require additional haemostatic agents during removal and tolerated the procedure well without any bleeding. LMWH was restarted 24 hours postremoval, and he continued with therapeutic anticoagulation levels and no bleeding symptoms. Repeat echocardiogram performed 2 weeks later demonstrated stable thrombus. He received 6 months of anticoagulation. Follow-up echocardiogram 3 months after discontinuation of anticoagulation demonstrated continued improvement with increasing organization of the thrombus. The patient has remained on emicizumab for routine prophylaxis without any need for bypassing agent treatment.

Central venous access devices are commonly needed in the treatment of children with haemophilia, particularly those undergoing ITI. The most common complication of CVADs in patients with haemophilia is infection, with rates of $0.66 / 1000$ catheter days. Thrombosis has historically been reported to be much less common, with an incidence rate of 0.056 per 1000 catheter days as derived from a meta-analysis of 48 studies. ${ }^{1}$ These rates are likely an underestimate of the true prevalence as prospective screening studies have found CVAD-associated thrombosis in up to $81 \%$ of patients, although the majority are subclinical in nature. ${ }^{2}$ These studies emphasize the asymptomatic, chronic course of most CVAD-associated thrombosis in haemophilia patients, the majority of which do not require treatment. Thrombosis risk may be higher in patients with inhibitors receiving ITI given the potential for synergy between an indwelling catheter, high dose FVIII and bypassing agents. ${ }^{3}$ Our patient had all of these along with the additional risk factors of recurrent infections and multiple line replacement procedures. He did not have additional risk factors such as obesity, family history of thrombosis or other thrombophilic risk factors. Co-administration of aPCC and rFVIla likely contributed to this patient's thrombosis. This strategy has efficacy for refractory inhibitor patients, but must be used with caution. The availability of emicizumab may provide opportunities to avoid it altogether.

No evidence-based guidelines exist for the use of anticoagulation in patients with inherited bleeding disorders. Few cases have been described in the literature in paediatric haemophilia and catheter-associated thrombosis, as patients typically are treated with catheter removal. The need for anticoagulation more commonly occurs in the treatment of adults with haemophilia given comorbidities including atrial fibrillation and atherothrombosis. The patient's bleeding phenotype influences anticoagulation considerations. This is particularly difficult in the case of a patient with an inhibitor, as $~ 10 \%-20 \%$ of bleeds may not fully respond to treatment with bypassing agents (BPAs). ${ }^{4} \mathrm{As}$ such, the risk of uncontrollable bleeding often outweighs the benefits of anticoagulation in this population. Despite our patient's severe bleeding phenotype with concurrent inhibitor, the critical nature and associated symptoms of his thrombosis demanded treatment. Initially, consideration was given to surgical thrombectomy as that would be the recommendation in a non-haemophilic patient, but ultimately anticoagulation was deemed to be a safer alternative. Serendipitously, 
the approval of emicizumab occurred shortly before our patient's thrombosis progressed. Systemic anticoagulation with concomitant emicizumab has not been previously described. The availability of emicizumab provided multiple benefits in this situation. Some literature suggests that trough levels of $>10 \%-15 \%$ should be targeted prior to anticoagulation. ${ }^{5}$ Recent data in patients on emicizumab prophylaxis reports FVIII activity by human reagent chromogenic assay to reach $\sim 20 \mathrm{IU} / \mathrm{dL}$ by week $5 .{ }^{6}$ Data from HAVEN 1 and 2, two of the pivotal clinical trials for emicizumab approval, clearly demonstrate decreased bleeding rates with use of emicizumab in haemophilia A with inhibitors compared to those on prophylaxis with BPAs. ${ }^{7,8}$ Additionally, its subcutaneous mode of administration allowed discontinuation of further procoagulant infusions through the port. The choice of $\mathrm{LMWH}$ was made based on its safety and efficacy in children, ease of laboratory monitoring, and lower bleeding risk compared to warfarin. ${ }^{9}$

Consideration of surgical thrombectomy raised many questions regarding appropriate laboratory monitoring. Classically, heparinization during bypass procedures is monitored using activated clotting times (ACTs), a point of care, contact activated whole blood coagulation test. Little is known about the effects of emicizumab on ACT although the prescribing information reports interference. Further complicating the situation, we were unable to obtain baseline ACT data on our patient given the need for urgent anticoagulation and possible effects of LMWH on ACT. There is very little in the literature about CPB in patients with haemophilia, and all cases except one ${ }^{10}$ that have been reported have been in non-inhibitor patients. Fortuitously, our patient responded to anticoagulation and the need for CPB was obviated. This case demonstrates effective bleed control, as well as continued improvement in thrombosis and associated symptomatology with concurrent anticoagulation and emicizumab. The dose of emicizumab was increased prior to surgery, which would lead to a higher peak level, though there is no data to support increased efficacy with this approach. No additional haemostatic therapy was required for port removal in the presence of therapeutic emicizumab. Although this particular scenario is rare, its real-life efficacy data in these types of settings will help inform emicizumab use, which is critical given its expanding indications.

\section{ACKNOWLEDGEMENTS}

A.C Weyand performed chart review, data collection, analysis and interpretation and wrote the manuscript. J.A. Shavit, A.L. Dorfman and S.W. Pipe performed data analysis and interpretation and wrote the manuscript.

\section{DISCLOSURES}

AC Weyand has acted as a paid consultant for Shire and Kedrion, JA Shavit has acted as a paid consultant for Bayer, Shire, CSL Behring, Spark therapeutics, Novo Nordisk and Octapharma. SW Pipe has received research funding from Shire and Pfizer, serves on clinical steering committees for BioMarin, uniQure and Bayer, and is a consultant to Shire, Pfizer, BioMarin, uniQure, Bayer, Roche/Genentech,
CSL Behring, Alnylam, Novo Nordisk, HEMA Biologics, Bioverativ, Catalyst Biosciences, DNARx and Spark Therapeutics and AL Dorfman has no conflicts of interest to disclose.

\section{ORCID}

Angela C. Weyand (iD https://orcid.org/0000-0003-2595-8541

Steven W. Pipe (iD https://orcid.org/0000-0003-2558-2089

Angela C. Weyand ${ }^{1}$

Adam L. Dorfman ${ }^{2}$

Jordan A. Shavit ${ }^{1}$

Steven W. Pipe ${ }^{1,3}$ iD

${ }^{1}$ Department of Pediatrics, Division of Hematology and

Oncology, University of Michigan, Ann Arbor, Michigan

${ }^{2}$ Department of Pediatrics, Division of Cardiology, University of

Michigan, Ann Arbor, Michigan

${ }^{3}$ Department of Pathology, University of Michigan, Ann Arbor, Michigan

Correspondence

Angela C. Weyand, Department of Pediatrics, Division of Hematology and Oncology, University of Michigan, Ann Arbor, MI.

Email: acweyand@med.umich.edu

\section{REFERENCES}

1. Valentino LA, Ewenstein B, Navickis RJ, Wilkes MM. Central venous access devices in haemophilia. Haemophilia. 2004;10(2):134-146.

2. Price Ve, Carcao M, Connolly B, et al. A prospective, longitudinal study of central venous catheter-related deep venous thrombosis in boys with hemophilia. J Thromb Haemost. 2004;2(5):737-742.

3. Silvey MS, Carpenter SL, Wicklund BM. The development of pulmonary embolus in patients with haemophilia $\mathrm{A}$ and high-titre inhibitors - a case series and review of the literature. Haemophilia. 2015;21(2):e134-136.

4. Astermark J, Donfield Sm, DiMichele Dm, et al. A randomized comparison of bypassing agents in hemophilia complicated by an inhibitor: the FEIBA NovoSeven Comparative (FENOC) Study. Blood. 2007;109(2):546-551.

5. Martin K, Key NS. How I treat patients with inherited bleeding disorders who need anticoagulant therapy. Blood. 2016;128(2):178-184.

6. Kiialainen A, Schmitt C, Adamkewicz J, et al.Pharmacokinetics and biomarkers in persons with haemophilia $\mathrm{A}(\mathrm{PwHA})$ receiving emicizumab every 2 or 4 weeks [abstract in conference proceedings]. In: European Association for Haemophilia and Allied Disorders; 2019 Feb 6-8; Prague.

7. Oldenburg J, Mahlangu JN, Kim B, et al. Emicizumab prophylaxis in hemophilia A with inhibitors. N Engl J Med. 2017;377(9):809-818.

8. Young G, Sidonio RF, Liesner R, et al. HAVEN 2 updated analysis: multicenter, open-label, phase 3 study to evaluate efficacy, safety and pharmacokinetics of subcutaneous administration of emicizumab prophylaxis in pediatric patients with hemophilia $\mathrm{A}$ with inhibitors [abstract no. 85]. In: ASH 59th Annual Meeting and Exposition; 2017.

9. Andras A, Sala Tenna A, Crawford F. Vitamin K antagonists or lowmolecular-weight heparin for the long term treatment of symptomatic venous thromboembolism. Cochrane Database Syst Rev. 2012;10:Cd002001. 2012;18(10):1570-4.

10. Langin M, Konrad M, Reichart B, Bauer A, Lison S. Acquired factor VIII inhibitor and cardiopulmonary bypass. J Cardiothorac Vasc Anesth. 2018;32(2):952-955. 